Revue des patrimoines

$37 \mid 2018$

Jardins collectifs : de l'abbé Lemire aux jardins d'insertion. Typologies - Expériences - Enjeux de conservation

\title{
L'architecture de la Seconde Reconstruction. Une épreuve de patrimonialité et de souveraineté
}

The architecture of the Second Reconstruction. A test of patrimoniality and sovereignty

Noël Barbe et Aurélie Dumain

\section{(2) OpenEdition}

Journals

Édition électronique

URL : http://journals.openedition.org/insitu/19834

DOI : $10.4000 /$ insitu. 19834

ISSN : 1630-7305

Éditeur

Ministère de la culture

Référence électronique

Noël Barbe et Aurélie Dumain, «L'architecture de la Seconde Reconstruction. Une épreuve de patrimonialité et de souveraineté », In Situ [En ligne], 37 | 2018, mis en ligne le 09 janvier 2019, consulté le 01 mai 2019. URL : http://journals.openedition.org/insitu/19834; DOI : 10.4000/ insitu. 19834

Ce document a été généré automatiquement le 1 mai 2019.

In Situ Revues des patrimoines est mis à disposition selon les termes de la licence Creative Commons Attribution - Pas d'Utilisation Commerciale - Pas de Modification 4.0 International. 


\section{L'architecture de la Seconde Reconstruction. Une épreuve de patrimonialité et de souveraineté}

The architecture of the Second Reconstruction. A test of patrimoniality and sovereignty

Noël Barbe et Aurélie Dumain

Inscrite dans le cadre de l'institution du bâti de la Reconstruction comme problème public $^{1}$ au regard d'enjeux climatiques mais aussi patrimoniaux, une recherche ethnologique a été menée en 2014 dans les communes d'Ammerschwihr (Haut-Rhin), de La Bresse et Gérardmer (Vosges) ${ }^{2}$. Loin d'une perspective d'inventaire au sens des politiques régionales du patrimoine ou de documentation historico-mémorielle, loin aussi d'une volonté de sensibilisation d'une population à la valeur patrimoniale d'une architecture - deux postures que nous avons rencontrées localement -, le problème posé par le travail d'enquête est celui des relations que des habitants d'aujourd'hui entretiennent avec les bâtiments reconstruits. L'enquête interroge particulièrement la question de la valeur qui leur est attribuée par ceux qui y vivent au quotidien, ainsi que la portée qui est donnée, au regard d'une volonté de valorisation matérielle et touristique de ce bâti, à ce qui se présente comme des expériences humaines singulières voire des leçons que des habitants entendent transmettre. Autrement dit, il s'est agi d'interroger l'actualité ${ }^{3}$ de l'architecture de la Reconstruction en tant qu'elle provoque toute une série de discours, de pratiques, de comportements et d'institutions. Mais aussi la situation faite dans le présent à la chaîne d'événements constituants de la destruction et de la reconstruction. 


\section{L'architecture de la reconstruction comme patrimoine, une version savante}

2 La problématisation de cette enquête nous confronte, dès le début de la recherche, à une version savante de cette architecture, la prédéfinissant comme un " patrimoine » à faire « reconnaître » par « la conscience collective ». Par un travail de pédagogie, entamé ou à réaliser, des élus, des techniciens, des architectes s'accordent sur la nécessité d'apprendre à des habitants, ici pensés comme aphones, à lire les qualités d'un style architectural. Les architectes et historiens en charge d'une étude bibliographique sur l'architecture de la Reconstruction exposent ainsi leurs intentions :

L'objectif est de faire percevoir progressivement leur valeur patrimoniale par la conscience collective, afin qu'elle puisse en assurer la protection et la transmission aux générations futures. [...] Reconnaître ce patrimoine, c'est reconnaître une période d'intense activité architecturale, théorique et pratique. [...] Ces bâtiments sont empreints de qualités qui méritent d'être mises en avant, ils peuvent être qualifiés de patrimoine. [... Ce ne sont] pas des architectures dont la qualité est facilement perceptible. Ils nécessitent une médiation pour être observés en euxmêmes, et requièrent des clés de lecture pour être compris entièrement ${ }^{4}$.

La conception du patrimoine empruntée ici, définie comme « l'ensemble des biens hérités des ascendants et conservés pour être transmis aux descendants $»^{5}$ procède d'une conception linéaire du temps allant du passé vers le futur ${ }^{6}$, imposant un «souci conservatif » - partagé pour l'heure par une « conscience minoritaire ${ }^{7}$ éclairée - à une " conscience collective " à faire advenir, par un travail de médiation qui se déroulerait sur deux plans : historique (soit une histoire matérielle à faire sur un bâti défini comme un patrimoine en soi et déjà là) et pédagogique (permettant d'apprendre à tout un chacun d'apprécier ses «qualités» formelles). Enfin, l'objet de la reconnaissance, i.e. une conversion patrimoniale par révélation d'un sens caché, est le travail d'architecture, une ville-œuvre ${ }^{8}$. Autour de ce constat, un " partage du sensible » au sens de Jacques Rancière est posé, définissant "en même temps un commun partagé » (ici un bâti en tant qu'il serait patrimonial à percevoir en tant que tel), et «la manière dont il se prête à participation " (ce que l'on peut y voir, en dire et y faire) « et dont les uns et les autres ont parts à ce partage $»^{9}$ (des habitants aphones et pour l'heure aveugles sur le sujet qui ont parts au fait d'être à éduquer par des experts). Ce constat est donc éminemment politique, au sens où il « porte sur ce que l'on voit et ce que l'on peut en dire, sur qui a la compétence pour voir et la qualité pour dire, sur les propriétés des espaces et des possibles du temps $»^{10}$.

4 À en suivre les ouvrages dirigés par Patrick Dieudonné sur les villes reconstruites ${ }^{11}$, cette approche patrimoniale et pédagogique participe de l'écriture d'une nouvelle histoire de l'architecture de la Reconstruction, passant du souvenir historique de villes détruites à un intérêt pour des villes reconstruites, donnant parfois lieu à des visites touristiques ${ }^{12}$. Cette réhabilitation et cette patrimonialisation de la Reconstruction, largement entreprises par des analystes de la planification, créent une "mémoire déséquilibrée » ou plutôt fragmentée de ces lieux, prise entre une histoire positiviste des formes d'un patrimoine en soi fondée sur l'analyse de « traces objectivées » d'une part, et d'autre part la réflexivité d'habitants, informée par des usages multiples, des remémorations plurielles d'un espace habité fait de traces incorporées, visibles mais aussi invisibles, chargé de représentations sociales et d'affects ${ }^{13}$. Proposer une troisième version de 
l'histoire et l'actualité de cette architecture demanderait de redistribuer les capacités ou de l'appréhender non seulement comme des dispositions matérielles mais aussi « des dispositions spatiales [...] et sociales qui fabriquent [...] une stratification d'ambiances ${ }^{14}$. Un autre partage du sensible est alors en jeu, ne consistant pas tant à réhabiliter l'affect contre le discours qu'à remettre en cause leur séparation.

\section{Comment redistribuer les capacités ? Tendre une épreuve de patrimonialité}

5 Face à cette double injonction, patrimoniale et pédagogique, cette enquête propose donc une autre problématisation : que peut-on apprendre des liens tressés entre les habitants et leur bâti ? Comment faire place à la pluralité des voix de population dans la désignation de ce qui fait patrimoine, mais aussi dans la définition du sens de l'espace dit public et de ce que l'on souhaite transmettre? Le cas de cette architecture récente nous confronte à une situation originale en matière de patrimoine, puisque, si la recherche en sciences sociales se concentre généralement sur l'étude des représentations et des usages des monuments et des dynamiques de monumentalisation « réussies $»^{15}$, qui transforment des bâtiments ordinaires en "lieux de mémoire », le résultat de ce processus est ici incertain ou peu stabilisé, puisque le statut de ces lieux serait justement à définir. Se situer au moment de cette épreuve de patrimonialité, tendre cette épreuve et la maintenir ouverte se présente comme une occasion pour éprouver les qualités patrimoniales de ce bâti, son statut dans la définition autochtone d'une historicité du lieu, mais surtout notre capacité à produire du patrimoine, du sens et l'avenir des espaces dits publics. Concrètement, les entretiens individuels et collectifs ${ }^{16}$ ont été l'occasion pour des habitants de faire passer les lieux par différentes épreuves destinées à leur reconnaître ou non une monumentalité et une valeur patrimoniale, une qualité artistique et architecturale, ou simplement à les envisager comme espaces privés et publics plus ou moins habitables ou vivables.

\section{Un patrimoine? Un monument ?}

6 À la question «L'architecture de la Reconstruction est-elle un patrimoine pour les habitants? », beaucoup d'entre eux ont répondu par la négative en convoquant un critère d'ancienneté : «c'est pas un patrimoine», parce que le patrimoine, c'est «l'ancien», «c'est trop moderne » ou « c'est trop tôt »: « je les vois comme les nouveaux bâtiments d'aujourd'hui, c'est les mêmes matériaux, sauf le côté vaste", nous dit-on à Ammerschwihr. Le patrimoine est situé ailleurs, dans les autres villages, moins détruits, dans les autres bâtiments de la commune ou dans les «restes", datant d'avant la destruction, comme l'ancien village de Gérardmer (fig. 1, fig. 2).

Figure 1

7 [Image non convertie]

Ammerschwihr, hôtel de ville reconstruit et sa place.

Phot. Noël Barbe, 2014. @ C Noël Barbe. 
Figure 2

8

\section{Ammerschwihr, partie ancienne de la ville.}

PHOT. NOËL BARBE, 2014. C C NOËL BARBE. passé des lieux, de prendre pied dans ce temps et de construire une profondeur historique du lieu : « Le petit point faible de La Bresse, c'est que... y'a pas de passé, y’a pas d'histoire [...] d'avant-guerre. [...] On ne retrouve pas les époques dans l'architecture.» Selon cette conception, la guerre comme événement destructeur agit comme une borne temporelle faisant le partage entre ce qui est patrimoine ou non. "On a perdu notre patrimoine ». Ce qui pourrait émarger au rang de patrimoine, c'est ce qu'il y avait avant la destruction et y a survécu, mais aussi ce qui fait signe de la vie d'avant et de l'événement qui l'a emportée : "les restes », les "ruines ", qui ouvrent sur un monde invisible et parfois imaginaire: «Quand je passe devant les ruines de l'ancien hôtel de ville d'Ammerschwihr, je pense toujours à comment vivaient les gens, mon père disait que les gens discutaient sur un banc ». À La Bresse, c'est un morceau du balcon de l'hôtel de ville détruit le 12 novembre 1944 (fig. 3), à Ammerschwihr ce sont aussi les restes de l'ancien hôtel de ville ou un jeu de panneaux urbains venant confronter ce qu'il y avait là et ce qu'on y voit désormais.

Figure 3

\section{[Image non convertie]}

La Bresse : au premier plan le balcon de l'ancien hôtel de ville, à l'arrière-plan l'hôtel de ville reconstruit. Phot. Noël Barbe, 2014. @ Noël Barbe.

Ce n'est pas plus un patrimoine au sens d'une typicité locale, un patrimoine alsacien ou vosgien. Ce disant, on regrette une perte d'identité du bâti : «Les immeubles [...] sont moins dans l'esprit alsacien des maisons à colombages ». « Ça pourrait être des bâtiments [...] transposés un peu n'importe où ». Cet esprit local se trouve alors incarné dans d'autres choses. Ainsi, le patrimoine, ce sont des objets « simples » qui ne relèvent pas du monumental, comme les beuhreux ${ }^{17}$, la schlitte ${ }^{18}$ (fig. 4), des éléments jugés caractéristiques de l'agriculture locale comme la vigne à Ammerschwihr, des éléments naturels comme le lac à Gérardmer, ou la cigogne.

\section{Figure 4}

12 [Image non convertie]

Tableau exposé dans l'hôtel de ville de Gérardmer.

PHOT. NOËL BARBE, 2014. (C) NOËL BARBE.

13 Ou une identité locale, qui se définirait par un devoir de conserver des ruines comme une « dette envers les ancêtres », soit un régime de domesticité. Celles-ci peuvent alors être rapprochées, dans le sens qui leur est donné, des monuments - aux morts, aux fusillés ou aux malgré-nous ; le lieu de rassemblement des Bressauds pour les déporter à Pforzheim en 1944, la maison où a habité " Miss Thérèse Bonney ", reporter de guerre, et la maison forestière d'Ammerschwihr, construite avec l'aide de la Fédération nationale des 
combattants et prisonniers de guerre en 1948 -, de la puissance rituelle de l'exode reconstitué des Bressauds. Mais ce sont aussi les figures du témoin ou de l'érudit local qui sont données comme incarnations patrimoniales, parce qu'elles restaurent la proximité avec un passé ; ou encore des situations morales, comme la souffrance liée à la guerre et la capacité à se relever du malheur et à manifester la puissance de la vie (fig. 5, 6, 7).

Figure 5

Enveloppe de don pour le groupement des sinistrés de La Bresse.

Phot. Aurélie Dumain, 2014. @ Aurélie Dumain.
15
Traces de projectiles sur l'église de La Bresse.

PHOT. NOËL BARBE, 2014. (C) NOËL BARBE. voit alors qualifiée de monument au sens de valeur historique destinée à maintenir des événements en mémoire. À cet égard, ce bâti est vécu comme un résultat de l'histoire, un " héritage », "C'est l'histoire qui a voulu ça ». Il fait trace de l'événement de la guerre : «Quelque part, c'est quand même un patrimoine », parce qu'«il est là » et qu'« il faut faire avec ». Comme la guerre, c'est un " patrimoine négatif $»^{20}$, « subi », «c'est l'histoire, on n'y peut rien ». Pour ceux qui ont vécu cette période ou dont les familles ont été touchées par sa violence, ce bâti provoque - à certains moments - l'irruption de l'événement traumatique invisible directement, des souvenirs qui, comme un spectre, reviennent sans cesse et hantent la lecture que l'on peut en avoir ${ }^{21}$. "S'ils sont là tels qu'ils sont actuellement, c'est parce qu'il s'est passé quelque chose. Mais, si on ne connaît pas l'histoire, [...] ça ne dit rien, si on ne creuse pas un peu ». Ce patrimoine est, par exemple, saisi comme un «témoin muet» de la guerre par un habitant de La Bresse, passionné d'histoire locale, qui, prenant appui sur une carte touristique actuelle, cartographie l'ensemble des drames subis par ses ancêtres, ouvrant alors un espace et un temps marqués par les bombardements, les incendies, la résistance, les dénonciations, les fusillades et l'exode.

Chargé de ces souvenirs, ce bâti est qualifié de "triste», blessé, parfois vécu comme seconde table rase ou destruction: "on peut regretter les choix qui ont été faits effectivement [...] de tout raser ». Une diversité de régimes d'attention coexiste toutefois, à la différence de ce passionné d'histoire locale qui n'y pense d'ailleurs pas tout le temps. Des touristes et une directrice de l'école avouent au contraire «ne pas y penser», «ne pas savoir », quand d'autres évaluent encore l'importance d'un « devoir de mémoire » 
avec l'idée de créer des marqueurs visibles de cette histoire ou bien au contraire un droit des habitants à «l'oubli ».

Dans ces conditions, parler de l'architecture de la Reconstruction s'apparente à accomplir un travail de deuil. Celui-ci est décrit comme non terminé, en particulier à La Bresse, où un cycle de la souffrance est représenté par les vitraux de l'église ${ }^{22}$ (fig. 8) et où des défenseurs de l'environnement décrivent, nouvelles destructions, des installations de ski qui continuent à « trouer », « miter » le paysage (fig. 9).

Figure 8

[Image non convertie]

Église de La Bresse, deux des vitraux de Gabriel Loire. Ce sont les destructions de 1803 et 1944 qui y sont évoquées.

PHOT. NOËL BARBE, 2014. ㄷ NOËL BARBE.

Figure 9

21 [Image non convertie]

HOHNECK, STATION DE SKI.

PHOT. NOËL BARBE, 2014. ㄷ NOËL BARBE.

Dès la Reconstruction, un travail de commémoration a été engagé, quand des habitants ont "sauvé » «le perron de la mairie » de La Bresse ou "les ruines de l'hôtel de ville » d'Ammerschwihr détruit en décembre 1944 et dont les restes sont classés monuments historiques $^{23}$ (fig. 10). Dans ces lectures historiques et sensibles du bâti, ces édifices, comme les ruines, se présentent enfin comme des leçons de résilience. "Que nous apprennent ces éléments considérés comme des traces de la guerre", avons-nous demandé ? "Laisser la ruine, ça donne à réfléchir quand même ", explique un villageois qui a dû reconstruire sa maison quand les cendres étaient encore chaudes; " y'avait une fois une guerre où tout était démoli. Et c'est important, pour que les jeunes sachent [...] qu'on a été victimes d'abord et qu'on s'en est sortis ; on a dû se battre. »

Figure 10

[Image non convertie]

Ammerschwihr, plaque commémorative.

PHOT. NOËL BARBE, 2014. (C) NOËL BARBE.

\section{Une architecture?}

Une épreuve esthétique et artistique est ouverte par la question: "est-ce une architecture? ? En contraste avec l'histoire lourde de la guerre, ce bâti est qualifié par une absence de prise singulière ${ }^{24}$ (c'est «lisse ", «ça n'accroche pas le regard»), d'éléments remarquables, qui suscite une certaine indifférence et une réponse négative : "C'est pas de l'architecture. » Les prises peuvent aussi être négatives, quand certains la qualifient de «moche ». «C'est pas beau, c'est pas fonctionnel, et il n'y a pas d'effort pour adapter à chaque village ; c'est le style Stoskopf, c'est pas un style alsacien ${ }^{25} »$. Ou encore, il n'y a « pas de cachet, de typicité ; c'est pas original ». Éprouver la grandeur d'un geste architectural passe donc par l'évaluation croisée de différents critères esthétiques: la 
définition d'un style singulier à l'architecte-artiste ou le respect d'une typicité par la reprise de motifs marqueurs d'une identité architecturale. La personnalité et les compétences de l'architecte sont également jaugées, par la reconnaissance de son œuvre à l'extérieur, mais aussi des critères fonctionnels dont la capacité des bâtiments à traverser le temps (fig. 11, 12, 13).

Figure 11 l'avènement de la "modernité » capitaliste. Certains se passionnent alors pour "une période d'intense activité architecturale ", à laquelle ils ont pris part, décrite comme une aventure humaine et économique : « une architecture innovante, sobre et adaptée » d'un point de vue fonctionnel, accompagnant la modernisation de la viticulture, du tissage. Des architectes sont même qualifiés de "visionnaires ", repensant l'organisation urbaine de Gérardmer, avec notamment la création d'un boulevard, défini comme une préfiguration des périphériques, chargé de fluidifier la circulation en vue de développer l'économie d'une ville balnéaire. C'est enfin une architecture d'urgence, le signe d'une époque, qui est décrite, marquée par une valeur d'usage et une morale du temps qui se formule: «c'est moche, ça n'a pas de style ", mais «c'est facile de critiquer après ", « il faut se replacer dans le contexte», "ils ont su répondre dans l'urgence aux besoins de logements », « on n'avait plus rien», « il fallait reloger », « il faut leur pardonner ». Autrement dit, l'attribution de la qualité d'architecture ne peut se faire que par un détour de situation, par inscription dans une période.

\section{Des espaces habitables?}

Ce bâti est-il habitable au sens de vivable enfin ? La compétence mobilisée pour évaluer une valeur d'usage et un confort est celle de l'entrepreneur qui a conduit les travaux, de l'habitant qui a lui-même dû faire des réparations, ou possède une connaissance familière ${ }^{26}$, routinière des lieux. À Gérardmer, beaucoup soulignent la qualité «moderne » de l'architecture (par exemple, la luminosité des grandes fenêtres synonyme de confort) et sa capacité à traverser le temps, informée par une conception hygiéniste reliée à 
Le Corbusier ${ }^{27}$. Mais, d'une manière générale, cette modernité, synonyme de confort des années 1950, est travaillée par un temps des générations, mise à l'épreuve par l'évolution du rapport aux énergies, aux circulations, aux modes de production et à l'évolution des modes de vie, quand les différentes générations ne vivent plus sous le même toit. La question du bilan énergétique de « ces grandes maisons vides » se présente alors comme un objet de préoccupation partagé par des habitants, des élus et des experts.

Comme toutes les maisons familiales et en particulier celles qui sont liées à des épreuves et à un travail des propriétaires, elles se présentent comme un patrimoine privé, un héritage familial, que les ascendants désirent transmettre. Mais c'est surtout la figure de l'héritage comme "charge " pour les descendants qui s'impose. Un viticulteur explique qu'avec l'évolution des règles de transmission du patrimoine, alliée au prix des maisons dans le vignoble, la maison devient pour son fils « une corde au cou », quand un autre s'effraie à l'idée des conséquences en termes de nouvelles contraintes pour les habitants que pourrait entraîner une éventuelle reconnaissance d'une valeur patrimoniale du bâti de la Reconstruction.

Sur un plan collectif, l'avenir de "grandes maisons vides » sans usages, sans repreneurs, car «trop grandes » et «trop chères », ou de "friches », qualifiées de "verrues », qui se multiplient dans les centres-bourgs définis comme "morts", est décrit comme "un problème ». « On n'est pas sorti de l'auberge », résume un acteur de la Reconstruction, se sentant mis devant/face à une double épreuve : il a fallu reconstruire et aujourd'hui, on a un nouveau problème. Faut-il garder ces maisons ou les détruire? « C'est du matériel, on n'est pas obligé de s'attacher ", lance un habitant, qui suggère la nouvelle destruction d'une « architecture d'urgence, qui n'avait pas de passé et par là-même n'a pas de futur ? [...] Il faut penser autre chose ». Quelques traces pourraient en être gardées pour les générations futures et les architectes à venir (fig. 14).

Figure 14

\section{[Image non convertie]}

La Bresse, hôtel de ville, dessin d'enfant, 2014.

PHOT. NOËL BARBE, 2014. @ C NOËL BARBE.

\section{Conclusion}

Devant/Face à la pluralité et la complexité des relations à ce bâti, ce sont d'abord les temporalités dans lesquelles il prend forme et s'articule qu'il nous fait saisir. Il vient briser le cours du temps et faire barrage à ce qui pourrait être une culture locale fondée sur la durée ou une histoire longue. Si la monumentalité est le point fixe à partir duquel se pensent les ruptures, les manières de se raconter et les origines ${ }^{28}$, alors l'architecture de la Reconstruction a bien un caractère monumental et son érection autorise une caractérisation de la localité, moins en termes de particularisme culturel que du point de vue d'une capacité à se reconstruire tant architecturalement que socialement ou psychologiquement. Le bâti de la Reconstruction échappe à la qualification de patrimoine, il ne peut incarner la durée. Ce qui a pu subsister ou être sauvé de l'avant prend corps dans d'autres artefacts: des restes de la ruralité d'avant ou dans ce qui manifeste sans cesse la répétition de la marginalité territoriale dans les conflits entre nations ${ }^{29}$. Ainsi des monuments aux malgré-nous. S'il est trace, c'est d'une situation, d'un moment, et par le 
détour de qualifications qui empruntent au registre de l'architecture. Enfin, face à l'avenir, il devient signe d'indécision mais porteur d'une capacité à faire face à l'effondrement d'un monde social.

Devant cette complexité, sommes-nous capables de faire place aux voix habitantes ${ }^{30}$ ? La réponse apportée par un élu à l'une des restitutions publiques de cette enquête, disant en ouverture du débat que, pour sa commune, «c'est un patrimoine, il n'y a pas de doutes ", indique la difficulté à accueillir une définition ouverte, plurielle, des visions concurrentes et éminemment sensibles de l'espace dit public ${ }^{31}$. Elle pointe aussi ce à quoi vise le travail d'institutionnalisation du patrimoine, soit le monopole de sa définition comme de ses narrations $\mathrm{s}^{32}$, latéralisant la portée des voix qui ne deviennent plus que du bruit, une justification ou un supplément d'humanité dans des dispositifs de médiation.

$\mathrm{Au}$ terme de cette enquête, il semble que l'expérience humaine et ses remémorations au sein de la population n'ont que peu de portée au regard des politiques publiques de droit commun liées à l'architecture, soit qu'elles s'attachent aux pures formes, soit qu'elles s'inscrivent dans une politique industrielle de développement touristique. Si l'on fait un pas de côté par rapport à ces deux injonctions, il reste une expérience singulière à transmettre. Elle n'est ni celle des formes architecturales ni celle de la neutralisation mémorielle. Plutôt une expérience humaine qui peut, au présent et au futur, être mobilisée face aux enjeux environnementaux et sociaux devant nous : l'expérience de la fin d'un monde social et sensible et une capacité à re-construire un monde qui, pourtant, n'est pas tout à fait celui que l'on a quitté. Une telle expérience possède incontestablement une dimension locale mais aussi, à côté d'autres, universelle. Organiser sa discussion et sa transmission, en faire provision pour le futur, autrement dit faire travailler différemment la question patrimoniale et celle de l'aménagement des espaces dits publics, en redistribuant les voix qui comptent, semble constituer un enjeu d'avenir.

\section{NOTES}

1. - C'est-à-dire comme un objet de préoccupation publique. On se reportera sur la question à NEVEU, Érik. Sociologie politique des problèmes publics. Paris : A. Colin, 2015 et, pour exemple, à GUSFIELD, Joseph. Contested Meanings. The Construction of Alcohol Problems. Madison : University of Wisconsin Press, 1996.

2. - Cette recherche a pris place dans un programme du parc naturel régional des Ballons des Vosges initié en 2014: "L'architecture de la Reconstruction est-elle un patrimoine pour le PNRBV?». D'autres axes de travail y sont développés, comme une mission d'expertise sur l'histoire, l'urbanisme et l'architecture de la Reconstruction. Au moment de l'enquête, l'un des auteurs de ce texte, Noël Barbe, était membre du conseil scientifique du parc naturel régional des Ballons des Vosges dont il a depuis démissionné.

3. - Au sens de FOUCAULT, Michel. "Qu'est-ce que les Lumières?» (1984). Dans FOUCAULT, Michel. Dits et écrits. II, 1976-1988. Éd. Daniel Defert et François Ewald. Paris : Gallimard, 2001, p. 1381-1397. 
4. - VARVENNE, Vanessa et TOUSSAINT, Aline. Architecture de la Seconde Reconstruction, un patrimoine pour le Parc? Synthèse bibliographique. Parc naturel régional des Ballons des Vosges, 2015, p. 95. Voir le site : https://www.parc-ballons-vosges.fr/agir/les-actions/architecture-de-laseconde-reconstruction-un-patrimoine-pour-le-parc/ [consulté le 27/12/2018].

5. - Ibid. La définition du terme "Patrimoine ", cité dans ce rapport, est issue du site du Centre national de ressources textuelles et lexicales (www.cnrtl.fr).

6. - Sur l'analyse d'une diversité de régimes d'historicité, voir HARTOG, François. Régimes d'historicité. Présentisme et expériences du temps. Paris : Seuil, 2003, ou PROUST, Françoise. L'Histoire à contretemps. Le temps historique chez Walter Benjamin. Paris : Éd. du Cerf, 1994.

7. - Ce «souci conservatif» porté par «une conscience minoritaire», capable d'imposer un «hommage dû au passé et un devoir à l'égard des générations futures », est informé - selon Daniel Fabre - par «le stigmate de la foule ignorante", souvent relié à une référence aux vandales de la Révolution, qui continue à nourrir l'indignation des institutions de conservation (FABRE, Daniel. «Introduction. Le patrimoine porté par l'émotion ». Dans FABRE, Daniel (dir.). Émotions patrimoniales. Paris : Éd. de la Maison des sciences de l'homme, 2009, p. 29-31).

8. - C'est aussi ce que défend ONIMUS-CARRIAS, Sophie. « Le patrimoine de la Reconstruction. Les villages de la poche de Colmar ». Patrimoines, 2008, 4, p. 84-92. Voir également RAOULX, Benoît. "Lectures et représentations du patrimoine de la Reconstruction, Le Havre et Caen ». ESO Travaux et Documents, 2005, 23, p. 17-24.

9. - RANCIÈRE, Jacques. Le Partage du sensible. Esthétique et politique. Paris : La Fabrique, 2000, p. 12.

10. - Ibid., p. 14.

11. - COLLOQUE INTERNATIONAL DES VILLES RECONSTRUITES, INSTITUT DE GÉOARCHITECTURE. Villes reconstruites, $d u$ dessin au destin. Actes $d u 2^{\text {e }}$ colloque des Villes reconstruites. Paris: L'Harmattan, 1994.

12. - FREY, Jean-Pierre. Dans ibid., vol. 2, p. 33-34.

13. - Ibid., p. 37.

14. - Appel à contributions de la revue Ambiances. «Ambiance et histoire de l'architecture. L'expérience et l'imaginaire sensibles de l'environnement construit » (2015).

15. - FABRE, Daniel. Op. cit.

16. - Le public d'enquête d'une centaine de personnes a été construit par un appel à "participation» du parc naturel régional via la presse locale et une information des mairies à leurs habitants. Si les communes ont constitué des listes de personnes potentiellement concernées par la thématique, des entretiens individuels et collectifs ont été proposés de notre part à des personnes évoquées dans l'enquête ou simplement rencontrées dans la rue.

17. - Muret en granit servant à délimiter les espaces pâturés.

18. - Traîneau en forme de luge servant au transport agricole et forestier.

19. - Cette fois au sens d'une valeur d'ancienneté repérée par Aloïs Riegl comme gagnant l'approche des monuments au $\mathrm{xx}^{\mathrm{e}}$ siècle, cette architecture, reposant sur une esthétique des ruines faisant traces de l'usure du temps qui passe, est considérée comme « trop moderne " pour constituer un patrimoine (RIEGL, Aloïs [1903]. Le Culte moderne des monuments. Son essence et sa genèse. Paris : Seuil, 1984).

20. - Sur la mine comme patrimoine négatif renvoyant à des événements et des images que l'on veut oublier, voir PERONI, Michel, et al. Historicité, localité et pratiques de patrimonialisation dans le bassin minier de la Loire. Rapport pour la Mission du patrimoine ethnologique, 1999, p. 14, ou WAHNICH, Sophie. «L'impossible patrimoine négatif ». Les Cahiers Irice, 2011, 7, p. 47-62

21. - Sur la spectrologie, voir DERRIDA, Jacques. Spectres de Marx [1993]. Paris : Galilée, 2006.

22. - Quatre des vingt-huit vitraux, œuvres de Gabriel Loire (1904-1996), représentent les quatre destructions de La Bresse, respectivement en 1466, 1635, 1803 et 1944.

23. - Voir le dossier: http://www2.culture.gouv.fr/public/mistral/dapamer_fr? ACTION=RETROUVER\&FIELD_1=TOUT\&VALUE_1=\&FIELD_2=cmer1\&VALUE_2=Ammerschwihr\&FIELD_3=cmer6\&VALUE_3=\&FIELD_4=C 
\%20protection\&VALUE_8=\&NUMBER=6\&GRP=0\&REQ=\%28\%28Ammerschwihr\%29\%20\%3aLOCA\% 2cPLOC\%20\%29\&USRNAME $=$ nobody \&USRPWD $=4 \% 24 \%$ $2534 \mathrm{P} \& \mathrm{SPEC}=9 \& \mathrm{SYN}=1 \& \mathrm{IMLY}=\& \mathrm{MAX} 1=1 \& \mathrm{MAX} 2=1 \& \mathrm{MAX} 3=50 \& \mathrm{DOM}=\mathrm{MH}$.

24. - Au sens de BESSY, Christian et CHATEAURAYNAUD, Francis. Experts et faussaires. Pour une sociologie de la perception. Paris : Métailié, 1995. Soit la « rencontre entre un dispositif porté par la ou les personnes engagées dans l'épreuve et un réseau de corps fournissant des saillances, des plis, des interstices » (p. 239).

25. - Charles-Gustave Stoskopf (1907-2004) est nommé architecte en chef de la Reconstruction dans le Haut-Rhin et travaille sur certains des villages de la poche de Colmar, lieu de violents combats durant l'hiver 1944, parmi lesquels Ammerschwihr. Il est porteur d'une réflexion sur l'esthétique alsacienne et sur les rapports entre une architecture vernaculaire, les exigences de la reconstruction et les besoins de l'époque. On se reportera à BOLLE, Gauthier. « Les enjeux d'une biographie ». Les Cahiers de la recherche architecturale et urbaine, 2014, 30-31, p. 183-192, et à Id. Un acteur de la scène professionnelle des Trente Glorieuses, de la Reconstruction aux grands ensembles: l'architecte alsacien Charles Stoskopf (1907-2004). Thèse en histoire de l'architecture et aménagement de l'espace. Strasbourg: université de Strasbourg, 2014. Ainsi qu'à DENIS, Marie-Noëlle. «La reconstruction des villages alsaciens après la Seconde Guerre mondiale ». Revue des sciences sociales, 2012, 47, p. 174- 179.

26. - Au sens du régime de familiarité analysé par THÉVENOT, Laurent. « Le régime de familiarité. Des choses en personne ». Genèses, 1994, 17, p. 72-101.

27. - Après la guerre, Le Corbusier est nommé architecte-conseil de la ville de Saint-Dié, proche de La Bresse et Gérardmer, pour laquelle il élabore un projet de reconstruction contesté, au final non retenu. Voir CLERICUZIO, Peter. "Le Corbusier and the reconstruction of Saint-Dié. The debate over modernism in France, 1944-46». Chicago Art Journal, 2010, 20, p. 47-71. Il a cependant construit la bonneterie Duval, détruite en grande partie par un incendie en novembre 1944.

28. - FABRE, Daniel. « L'ethnologie devant le monument ». Dans FABRE, Daniel (dir.). Domestiquer l'histoire. Ethnologie des monuments historiques. Paris : Éd. de la Maison des sciences de l'homme, 2000, p. 1-29.

29. - Voir RAPHAËL, Freddy et HERBERICH-MARX, Geneviève (dir.). Mémoire de pierre, mémoire de papier. La mise en scène du passé en Alsace. Strasbourg : Presses universitaires de Strasbourg, 2002.

30. - Sur ce sujet, s'agissant du Havre, voir GRAVARI-BARBAS, Maria et RENARD, Cécile. «Une patrimonialisation sans appropriation? Le cas de l'architecture de la Reconstruction au Havre». Norois, 2010, 217, p. 57-73.

31. - En d'autres termes, et pour reprendre l'enjeu d'un autre "partage du sensible » fixé en préambule, l'enquête en sciences sociales associant les populations permet-elle de faire « du bruit » ou de faire entendre des voix habitantes comme « du discours » ayant autorité en matière de gestion des affaires de la cité ? Voir «Le partage du sensible. Entrevue avec C. Palmiéri ». Revue d'art contemporain ETC, 2002, 59, p. 34. Ou bien ne relève-t-elle que de la vaccine de Barthes : «On inocule un peu de progrès - tout formel d'ailleurs - à la tradition, et voilà la tradition immunisée contre le progrès : quelques signes d'avant-garde suffisent à châtrer la véritable avant-garde, la révolution profonde des langages et des mythes » (BARTHES, Roland. CEuvres complètes. Éd. Éric Marty. Paris : Seuil, 2002, I, 1942-1961, p. 564-565).

32. - Nous employons institution dans le sens de BOLTANSKI, Luc. De la critique. Précis de sociologie de l'émancipation. Paris : Gallimard, 2009. Soit un être sans corps à qui est déléguée la tâche de dire ce qu'il en est de ce qui est. 


\section{RÉSUMÉS}

Installant d'entrée de jeu l'architecture de la Reconstruction dans un régime d'indécision axiologique, ce texte interroge notamment l'attribution - ou son absence - d'une valeur patrimoniale à cet objet par ceux qui entretiennent avec lui des relations de familiarité, parce qu'ils y déroulent leurs vies au quotidien, au passé mais surtout au présent, dans trois communes - Ammerschwihr (Haut-Rhin), La Bresse et Gérardmer (Vosges) - où nous avons mené enquête. Sans grand étonnement, l'argumentation dominante, portée par une lecture savante et une volonté institutionnelle de valorisation touristique et économique en régime industriel (Boltanski), fait de ce bâti un ensemble patrimonial, le rapportant en particulier à une histoire des formes ou instituant la ville comme œuvre. Pour leurs familiers, ces villes reconstruites sont caractérisées par une monumentalité dans la mesure où leur architecture peut être le point fixe à partir duquel peuvent se penser des ruptures temporelles ou s'articuler les manières de se dire. L'architecture, comme la stèle commémorative, autorise alors la caractérisation de la localité parce qu'elle incarne justement une capacité à la reconstruction, certes du bâti, mais essentiellement des êtres. Aussi échappe-t-elle à la spécification patrimoniale parce qu'elle laisse échapper le temps comme durée. Enfin, question est posée de la distribution des pouvoirs d'asserter la valeur (Lordon) au regard du peu de portée d'une telle caractérisation dans les politiques publiques liées à l'architecture et à la patrimonialisation.

\section{INDEX}

Mots-clés : architecture de la Seconde Reconstruction, axiologie patrimoniale, épistémologie politique, formes de présence du passé, familiarité monumentale

\section{AUTEURS}

\section{NOËL BARBE}

Anthropologue, Institut interdisciplinaire d'anthropologie du contemporain CNRS-EHESS noel.barbe@cnrs.fr

\section{AURÉLIE DUMAIN}

Sociologue, Centre Max Weber/Politiques de la connaissance, Lyon dumainaurelie@gmail.com 